\title{
Developing A Lessons Learned Database for NCDOT Projects Using Design for Six Sigma (DFSS) Approach
}

\author{
Siddharth Banerjee ${ }^{a^{*}}$, Edward J. Jaselskis, Ph.D, P.E. , Abdullah F. Alsharef ${ }^{\mathrm{a}}$, Clare E. Fullerton, \\ P.E. ${ }^{c}$, Alyson W. Tamer, P.E., CPM ${ }^{\mathrm{d}}$ \\ ${ }^{a}$ Graduate Research Assistant, North Carolina State University, Raleigh, NC, 27695, USA \\ ${ }^{b}$ E.I. Clancy Distinguished Professor, North Carolina State University, Raleigh, NC, 27695, USA \\ ${ }^{c}$ Value Management Program Engineer, North Carolina Department of Transportation, 1020 Birch Ridge Dr, Raleigh, NC, 27610, USA \\ ${ }^{d}$ State Value Management Engineer, North Carolina Department of Transportation, 1020 Birch Ridge Dr, Raleigh, NC, 27610, USA
}

\begin{abstract}
Valuable lessons and best practices learned from construction projects often fail to get transferred to future generations due to the lack of a formalized process. This perpetual problem gives rise to the need of imparting fresh training to new inductees once the aging workforce retires or in the event of turnover in an organization. This paper aims to facilitate the process of disseminating knowledge gained in the transportation area by developing a lessons learned database named Communicate Lessons, Exchange Advice, Record (CLEAR) under the auspices of the North Carolina Department of Transportation (NCDOT). A Six Sigma approach to Identify, Define, Develop, Optimize, and Verify (IDDOV) was used to develop the CLEAR database. The IDDOV model in Design for Six Sigma (DFSS) is a five-step process in designing efficient and robust new systems. The first phase involved conducting interviews with end-users as well as reviewing existing data within NCDOT data repositories such as claims and supplemental agreements data to better understand challenges and issues in need of resolution. With this information as basis, an outline for web-based database was created from which feedback was solicited. After taking into consideration all preferences from these end-users, the web-based database was fine-tuned to reflect all comments and suggestions gathered by the research team. This database is currently being populated from ongoing pilot projects. In the final stage of this project, the CLEAR database will be launched throughout the state on all ongoing projects. These five project phases reflect the principles of the IDDOV method of Six Sigma which provides the foundation for developing this database. Findings from this study will help NCDOT to institutionalize knowledge and improve project cost variations and schedule predictability. The success metrics include anticipated reduction in number of claims, claim amounts and increased coordination among staff within NCDOT.
\end{abstract}

(C) 2019 The Authors. Published by Budapest University of Technology and Economics \& Diamond Congress Ltd.

Peer-review under responsibility of the scientific committee of the Creative Construction Conference 2019.

Keywords: Knowledge management; Lessons learned; Organization efficiency; Six Sigma; Web-based database

\section{Introduction}

Construction is a complex process involving methods and activities unique to a project that are seldom repeated on other projects in the same sequence. Construction companies constantly strive to improve their workflow by preparing

*Corresponding author: 222 Mann Hall, Department of Civil, Construction, and Environmental Engineering, North Carolina State University, Raleigh, NC - 27695, USA. Author email: sbaner22@ncsu.edu 
Banerjee, et al. / Proceedings of the Creative Construction Conference (2019) 039 https://doi.org/10.3311/CCC2019-039

robust schedules and periodically documenting project progress. Despite having sound procedures in place, companies still face issues that can lead to delays and claims, thereby causing financial loss and/or loss in reputation. One of the primary reasons for this is failing to document valuable knowledge learned by the personnel in their work. A lot of useful knowledge gained through years of experience goes waste if not documented properly and at the right time. This is especially true in organizations with high turnover or older personnel retiring from their positions, giving way to younger generations. Although the younger generations may be full of zeal in finishing tasks assigned to them, lack of access to previous experience may devoid them of the ability to make sound decisions. The concept of lessons learned systems (LLSs) has been gaining importance and are being deployed to utilize important knowledge gained over time. The greatest advantage of having a good LLS is to reduce rework and put to practice what worked well or did not work very well on a project. The Project Management Institute (PMI) defines lessons learned as the learning gained from the process of performing the project [1].

This paper describes an effort to design and implement a lessons learned database throughout North Carolina Department of Transportation (NCDOT). Additionally, NCDOT is currently facing turnover issues like any other organization and there is an urgent need to document lessons for later use. Lack of formalized processes compounds the problem and thus the lessons learned database will assist personnel to revisit past experiences rich in knowledge and bring in organizational changes to improve processes within the organization.

The proposed lessons learned database will be an internal only database that is mainly intended for personnel associated with all project phases within the construction domain in NCDOT. There are 14 divisions throughout the state. A Design for Six-Sigma (DFSS) approach for this project has been proposed which can be considered as novel in this field of research since previous research on designing lessons learned databases have overlooked this approach. A DFSS model allows for robust systems to be designed right from the inception till its design complete. This project makes use of the Identify-Design-Develop-Optimize-Verify (IDDOV) model of DFSS to design this database.

Design for Six Sigma (DFSS) methodology is a systematic and disciplined problem preventing approach which is widely used to design robust engineering systems. There are many models that utilize DFSS for generic technology development such as $\mathrm{I}^{2} \mathrm{DOV}$ (Invent, Innovate, Develop, Optimize, Verify), CDOV (Concept, Design, Optimize, Verify), IDDOV (Identify, Define, Develop, Optimize and Verify); DMADV (Define, Measure, Analyze, Design and Verify) to name a few [2]. While the above-mentioned models have their own benefits and drawbacks, the research team utilized the concepts of the closed loop IDDOV model that starts and ends with the customers. The actual scale of implementing this IDDOV model is not the same as used in the manufacturing or related sectors but drawing inspiration from them. Fig. 1. depicts pictorially the five steps of the IDDOV model as applied to the CLEAR database creation. The research team explored various models and selected the IDDOV model most suited to design the database and to build an error-free robust lessons learned database. Beyond a doubt, DFSS can be considered a natural fit for software development process (SDP) owing to similar approaches [3].

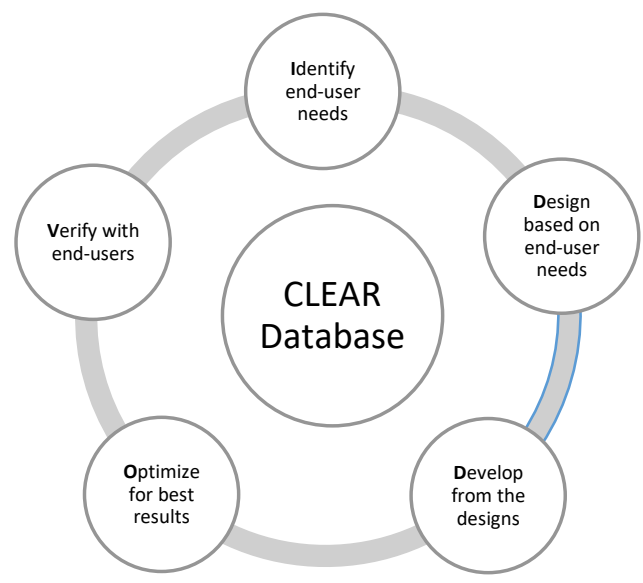

Fig. 1. Six-Sigma DFSS Approach Using IDDOV Applied to CLEAR Database 
Banerjee, et al. / Proceedings of the Creative Construction Conference (2019) 039 https://doi.org/10.3311/CCC2019-039

The Design for Six Sigma (DFSS) is a part of Six Sigma philosophy that is applied while designing novel systems. Sokovic and Pavletic [4] provide the following definition for DFSS:

"DFSS methodology is a systematic and disciplined approach to product or process design, including all organizational functions from the early beginning, with the objective to design things right from the first time".

Six Sigma concepts can be applied at any phase in a project, depending on its need and the final requirements. The Define, Measure, Analyse, Improve, and Control (DMAIC) model is the most popular model within Six Sigma [4] [5]. As can be seen from Fig. 2., the DMAIC approach is best suited to improve existing processes. In contrast, the IDDOV model of DFSS is best suited for novel design requirements. Since the NCDOT lessons learned database is being created from the outset, the IDDOV model served in the best interests of the research team. The five phases of IDDOV as applied to this project are described in section 2.2.

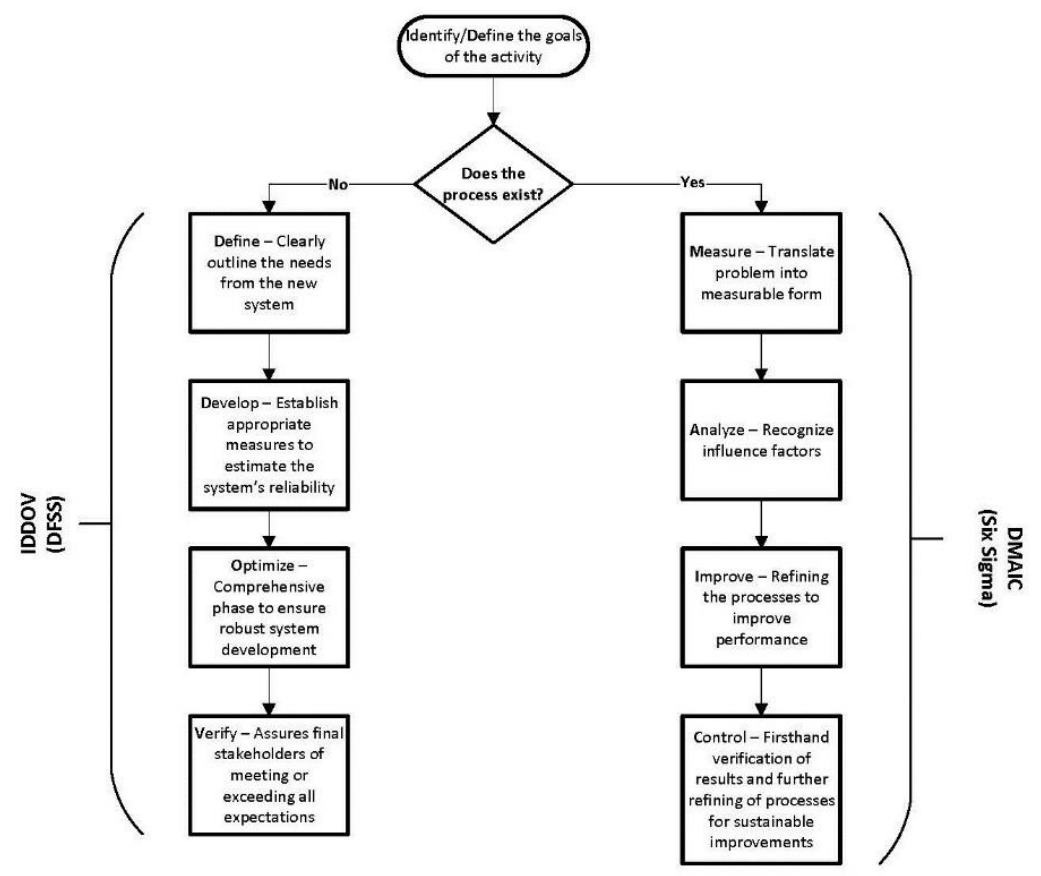

Fig. 2. Typical decision approach for traditional Six Sigma (DMAIC) and DFSS (IDDOV)

\subsection{Background}

Currently, there is no formal mechanism in place within NCDOT for sharing useful knowledge among design, construction, and maintenance personnel and within the fourteen divisions throughout the state. There is a silo-type mentality and hence very limited communication on project successes and failures among people exists. This gives rise to two problems. First, there is in no way to inform the other divisions/personnel about a particular method or product other than a direct contact in a conference or telephone. Owing to immense work pressures, there is little time for personnel to convey successes and failures to each other. Second, the very fact that something can be done better by following certain steps remains with each person and is not effectively conveyed to others. Thus, there is a need to establish a method of collecting lessons learned to share valuable knowledge among design, construction, and maintenance staff. This information will help improve design guidelines, best practices, revise specifications and bring to light innovations that have taken place on projects that could be used throughout the NCDOT organization. Effectively sharing information of this nature can reduce project costs and improve project delivery. 
Banerjee, et al. / Proceedings of the Creative Construction Conference (2019) 039 https://doi.org/10.3311/CCC2019-039

This project involves the collection and dissemination of both lessons learned and best practices during the preconstruction phase, execution phase (considering detailed design and construction), and maintenance and operations - essentially covering all aspects of the project lifecycle. A user-friendly, web-based accessible database in SharePoint will be used to both collect and communicate lessons learned and best practices. The database would be sortable by major trends for the various groups within NCDOT.

\subsection{Previous Work}

Organizations have now realized that there is a need to store and retrieve useful lessons. Previous research on this topic has explored various approaches for lessons learned experiences in the construction industry [6]. Additionally, the Kentucky Transportation Cabinet funded a study to develop a constructability lessons learned tool used during the design phase to improve project outcomes [7]. Fong and Yip [8] measured the level of readiness of construction professionals in Hong Kong about being open to implement lessons learned systems within their organizations. They utilized a survey instrument to solicit information from construction professionals to learn about the current practices of recording and retrieving lessons learned in organizations within Hong Kong and to understand the critical design aspects for a lessons learned system from an end-users perspective. One of their research finding was that construction personnel did not prefer to record lessons while the project was ongoing, which can lead to important knowledge being lost. This is one of the pitfalls the current research has taken note of and aims to avoid.

Lessons learned exercises have been an integral part of improving processes in an organization. It has been recognized as one of the 17 best practices identified by the Construction Industry Institute (CII) for achieving enhanced project performance [6]. Lessons learned act as a valuable constructability resource to planning and design teams to help identify potential problems in advance and thus be proactive in mitigating possible schedule and cost overrun issues.

The Construction Industry Institute (CII) report on lessons learned [9] acts as an invaluable resource in the field of knowledge management. This report highlights the three main phases of a lessons learned exercise as collection, analysis, and implementation. Establishing the right culture and upper management support is also essential to establishing a successful lessons learned program. The authors also suggest that in an organizational structure, knowing what to document and where could impact the effectiveness of a designed lessons learned tool. Most organizations have now started to realize the full potential of a lessons learned program within their organizations. For instance, NASA has both a public lessons learned system (PLLS) as well as internal lessons learned information system (LLIS). The U.S. Army Construction Engineering Research Laboratories (CERL) uses DrChecks, which utilizes client-server architecture for comment sharing among various parties pertaining to design documents over the web.

The Indiana Department of Transportation (INDOT) was an early adopter using a lessons learned database. In consultation with researchers McCullouch and Patty [10] at Purdue University, a series of interviews was conducted with personnel at INDOT to improve coordination between the design and construction teams aiming to achieve a better constructability review program. A windows-based constructability lessons learned software application was developed using visual basic. Folio Views is the software that contains the Constructability Lessons in text form and is also used to store, index and retrieve the lessons.

A similar research activity was performed jointly by Kentucky Transportation Center and University of Kentucky. Goodrum et al. [11] surveyed resident engineers, contractors, and consultants to obtain an initial understanding of what they envisioned to be a perfect lessons learned database. The team came up with relevant data fields to be populated in a web-based lessons learned database which could accept files both in text as well as image format. Each user associated with the database was classified into three categories - end user, gatekeeper, and administrator with each of their functions clearly stated. The database was structured into two parts - one for users to enter new lessons learned and the other for storing and retrieval of cleaned up lessons. MS Access was implemented for data storage and retrieval while MS FrontPage was used to accept lessons learned input from users. The database also had provision to search for specific terms within the database fields to yield specific results helpful for design teams during a constructability review. The MS Access database however had a capacity of just 2000 rows and the plan was to link this database with an Oracle database for wider reach [11]. However, this effort did not seem to be very successful as the lessons learned database ran a high risk of being defunct once the 2000 rows limit was reached in case of failed integration. 
Banerjee, et al. / Proceedings of the Creative Construction Conference (2019) 039

https://doi.org/10.3311/CCC2019-039

\section{Research Methodology}

\subsection{Overview of Six Sigma Philosophy}

The term Six Sigma was coined by engineers working in Motorola during the ' 80 s and has since been adopted by other major corporations such as Toshiba, General Electric, Ford Motor Company. The six-sigma philosophy is a statisticsbased concept that is widely used in multiple business sectors these days. It focuses on better understanding of enduser requirements, improving business systems throughout the organization by reducing the amount of errors and continuous improvement of all organizational business systems. The ultimate benefits of adopting six-sigma model are improved quality and delivery of products, development of robust processes, and sustained competitive advantage [5].

Six Sigma philosophy aims at minimizing the number of defects and reducing variation in processes. The Greek letter Sigma signifies the standard deviation for a population. In the context of Six Sigma, this standard deviation is directly proportional to the process variation. That is, the Sigma value reduces with reduction in process variation and vice versa. Mathematically, Six Sigma aims at achieving 3.4 defects per million opportunities (dpmo) which is near perfection, whereas a three Sigma process aims at achieving about 66,807 dpmo [12]. Over the years, there have been many versions of Six Sigma that were conceptualized by organizations based on their requirements. The Define, Measure, Analyze, Improve, and Control (DMAIC) is widely considered as one of the most popular Six Sigma datadriven quality strategy to improve processes and for quality control in an organization. Thus, it becomes important to distinguish between Six Sigma and Design for Six Sigma (DFSS). While Six Sigma process improvement is a method for improving process performance and capability without process redesign, DFSS is a Six Sigma strategy that focuses on the early stages of a product/process life cycle [13]. Table 1 below shows the differences between Six Sigma and DFSS as applied to a business model for various associated business elements required by an organization. At this point, it is reiterated that this project utilizes the beliefs of the DFSS model but does not employ any associated statistical measures and/or related calculations. The five phases of IDDOV as applied to the CLEAR database is as described below.

Table 1. Differences between Six Sigma and DFSS [14]

\begin{tabular}{ccc}
\hline Element & Six Sigma & DFSS \\
\hline Focus & Existing process & New process \\
Goal & Reduce variation & Reduce variation and optimize performance \\
Action taken & Analyze & Design \\
Best suited for & Maximizing current process & Developing new products \\
Major effect is on & $\mathrm{C}_{\mathrm{P}}$ (reducing variation) & $\mathrm{C}_{\mathrm{Pk}}$ (centering within customer requirements) \\
\hline
\end{tabular}

\subsection{IDDOV Model of the Database}

\subsubsection{Identifying needs and understanding trends}

This is the first step in the process and includes the necessary steps to be taken to form an initial structure of the database and to collect information about end-user's database requirements. The research team prepared an interview guide containing a list of questions asking the respondents about their preferences for a lessons learned database and to understand the current trends within NCDOT. The current trends imply the existing practices of recording data pertaining to claims, supplemental agreements, and other useful relevant data at NCDOT. The contact details of each potential respondent/end-user was provided by the NCDOT Value Management office. Since this process involved human interaction, an Institutional Review Board (IRB) approval was required from the appropriate university authority prior to acquiring information. An interview request was sent by email to 66 potential respondents at the 
Banerjee, et al. / Proceedings of the Creative Construction Conference (2019) 039

https://doi.org/10.3311/CCC2019-039

NCDOT. By the end of this phase, 32 interviews were conducted with 46 personnel with a total of 813 years of work experience. Most of these interviews were conducted over the telephone, but a few were performed in-person.

\subsubsection{Defining the Database}

After the research team was able to gather information on the trends and end-user requirements for the database, a qualitative analysis was performed to come up with the necessary database fields. It was important to not miss out on any relevant information and hence there were at least two sets of entries for each interview in Microsoft Excel sheets. At the end of the interviews, both the Excel sheets would be carefully investigated to ensure that all data had been captured and documented properly. The initial design of the database had three segments. The first segment captured basic respondent information. The second segment captured details about the lesson or the best practice with a provision to attach relevant files and images to augment information. The final segment included details regarding the project such as project number, contract number, project type, claims and supplemental agreement information.

\subsubsection{Developing the Database}

The third phase of the project involved developing the lessons learned database using the designs arrived at using the information gathering phase from end-users. Since this is an internal-only database and developmental information is accessible to NCDOT users alone, the Information Technology department within NCDOT (NCDIT) was tasked with developing the web-based database on a SharePoint portal using Microsoft Access. This combination was chosen because NCDOT currently has an active SharePoint portal for its normal operations and hence a preferred option by the end-users. The research team is currently working towards obtaining lessons learned files from the respondents. At this time, 29 lessons learned files were collected from 15 personnel contacted in the first phase. These files were submitted to the NCDIT to be populated in the newly developed pilot database. Additional respondents are being contacted to solicit more lessons learned. Once all the lessons are entered into the pilot database, the research team plans to visit a few pilot projects currently being constructed to check for the possibility of collecting more lessons from site engineers, inspectors, resident engineers, and other site personnel.

\subsubsection{Optimizing the Database}

The CLEAR database is being created to cater to all the 14 divisions of NCDOT and has a few functionalities that draw upon information from other central databases such as HiCAMS, a repository for NCDOT contract data. During the data gathering phase, the team identified that NCDOT personnel did not want to spend more than 5 minutes entering a lesson or searching for relevant ones. Hence, it became imperative to streamline information such that accurate information can be entered/accessed in a timely fashion. Additionally, since large amounts of space in the form of images and files and attachments can be expected for each lesson entered, careful attention needs to be given to allocate adequate space in the database. Thus, the need to optimize the database periodically based on the development in the previous stage. The KyDOT lessons learned database was initially designed for 2000 entries and thus had a huge risk of running out of space [11]. During a formal conversation with one of the engineers that worked with the Kentucky Transportation Center revealed that their database ran out of space within a year or so of its deployment once all the 2000 rows got filled. This fact was noted by this research team to avoid repeating the same mistake.

\subsubsection{Verify for completeness}

After all the previous steps have been performed, the database will be reviewed and tested by end-users to provide feedback regarding their satisfaction of the new lessons learned database. These comments will be collected and given to the IT team at NCDOT for possible improvements. In short, the team will verify the completeness of the system and rectify any shortcomings. All the five steps will be then repeated in an order till the gatekeeper finalizes the wholeness of the database as can be seen from figure 1. The gatekeeper would be responsible for ensuring the completeness of submitted lessons and for final uploading of these lessons into the lessons learned database. The Value Management team at NCDOT will act as the gatekeeper. 
Banerjee, et al. / Proceedings of the Creative Construction Conference (2019) 039

https://doi.org/10.3311/CCC2019-039

\section{Results and Future Work}

Currently, the research team is in the process of collecting lessons to populate the database. To date, the research team has been able to gather and handover to NCDIT 29 lessons to be entered into the pilot database. Appendix A shows a template for the lessons solicited from the end-users. The database fields were arrived at based on the initial information gathering phase. The database requirements from the end-users are as summarized below:

\subsection{Database requirements}

\subsubsection{User friendly}

- Less typing - The respondents looked forward to having more of drop-down menus to select their answers rather than typing it, as it would save some time and effort. This was however challenging for the research team but strived to bring all possible options as a drop-down menu.

- Keep data entry simple and less time consuming - Most of the respondents also mentioned that owing to multiple tasks throughout the day, it would not be possible for them to spend more than five minutes on entering/retrieving lessons.

- Avoid double data entry - Currently, work related details are entered in the engineer diaries as well as HiCAMS - a central repository to store information related to contract administration, claims, supplemental agreements. Thus, it was important to devise a strategy so that respondents did not need to enter data multiple times.

\subsubsection{Easily searchable}

- Display relevant and specific results - So as to not put off the respondents, it was crucial that the search results displayed were most relevant and suited to the requirements based on the search criteria. There is a risk of non-usage of the database if appropriate search lessons are not displayed which the user is searching for.

- Use appropriate filters/tags - To obtain accurate search results, users have an option to narrow down search results based on pre-defined criteria such as date observed, keywords, project type. The more information the user inputs as search condition, the better the search results will be.

\subsubsection{Other desired features}

- Upload images/pdf/email files - As they say a picture speaks a thousand words. Being able to upload an image file or other relevant attachments will provide better understanding of the issue and the solution taken to resolve the issue.

- Provide an impact rating (based on cost and time) - Provision to have impact ratings based on the project cost and schedule bearing direct influence on the lesson being entered. A Likert scale was mooted for rating both cost and schedule variations. This rating will be critical to search for highly meaningful impactful lessons.

- Create automatic trigger to complete a lesson learned on projects that experience claims or supplemental agreements above a certain threshold value

\subsubsection{Training}

- The lessons learned database can act as a training resource for fresh recruits to look up necessary lessons using the search functionality.

During the information and database requirements gathering stage, one of the key issues facing most NDCOT projects pertained to utility relocations/removal and coordination. As a result, the academic research team was requested to summarize all key findings from the interviews pertaining to utilities and submit this information to the Value Management Office for development of a special utility guide. This was validated through our interviews as well as data on claims on all NCDOT projects that was provided by the Value Management office. This gave rise to another research idea looking into the leading indicators of claims arising due to utilities which is currently being performed 
by the same research team. It is also planned to continue collecting and populating the pilot database and prepare for an organization wide rollout of the database around July 2019. To ascertain the success of the lessons learned database, the success measures would involve increased coordination among design, construction, and maintenance personnel. Additionally, there should be a decrease in the number of claims, supplemental agreements arising in construction projects, in addition to observing reduction in the amounts associated with them.

\section{Conclusions}

The lessons learned/best practices database within NCDOT would facilitate better coordination between design teams and construction teams. The overall aim of creating this database would be to achieve superior design performance and thus reduce the frequency and impact of change orders, achieve enhanced cooperation, and ultimately accomplish improved operational performance. Lessons learned are an effective way to document and retrieve wisdom gained from previous projects and to apply these learnings in future projects to attain best practices. Project teams are dynamic and seldom repeat themselves in different projects. Also, there are possibilities that the aging workforce will retire before their knowledge can be documented. In either case, there would be a significant amount of wisdom that would be lost if they are not documented in a proper lessons learned/best practice database. This will provide scope for the young generation to take cue and put into practice these lessons learned to realize desired project goals. Project teams across departments at the NCDOT will greatly benefit from utilizing this rich and robust knowledge database. In the long run, the CLEAR database will assist NCDOT to adjust future cost estimates, update standards, and change policies to continuously strive to be an effective and efficient organization to the public.

\section{Acknowledgements}

This research is supported by North Carolina Department of Transportation. We are thankful to all the NCDOT personnel who provided insight and expertise that greatly assisted the research, although they may not agree with all the interpretations/conclusions of this paper.

\section{Appendix A. Sample Lessons Learned/Best Practices Template}

CLEAR Lessons Learned/Best Practices
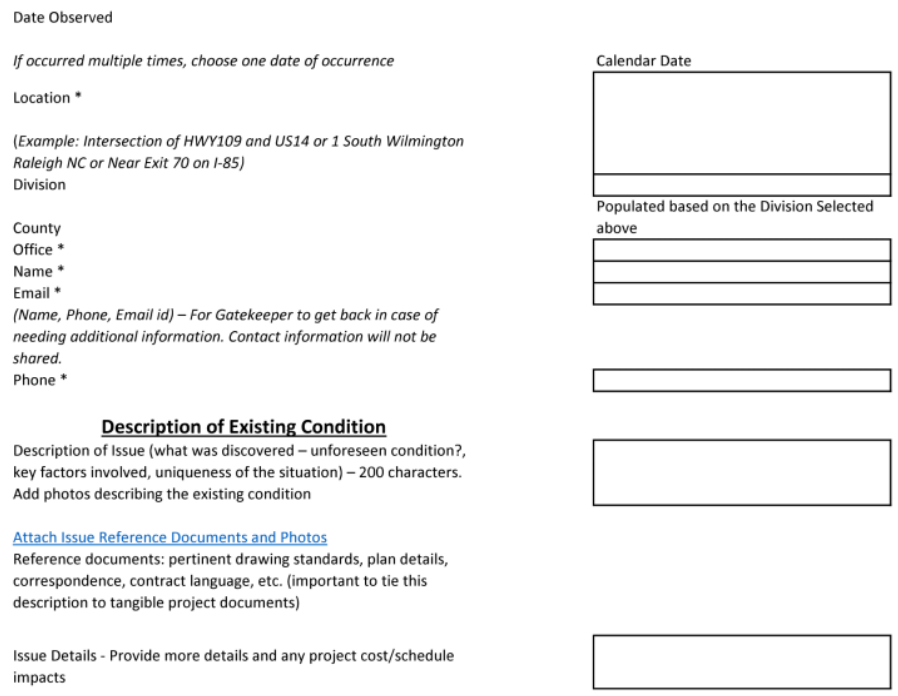
Banerjee, et al. / Proceedings of the Creative Construction Conference (2019) 039

https://doi.org/10.3311/CCC2019-039

References

[1] Project Management Institute, A guide to the project management body of knowledge (PMBOK®), 3rd ed., Newtown Square, PA: Project Management Institute, 2004, p. 363.

[2] M. Hu, J. M. Pieprzak and J. Glowa, "Essentials of Design Robustness in Design for SixSigma (DFSS) Methodology," in SAE 2004 World Congress \& Exhibition, 2004. https://doi.org/10.4271/2004-01-0813

[3] D. Grant and E. A. Mergen, "Towards the use of Six Sigma in software development," Total Quality Management, vol. 20, no. 7, pp. 705-712, 2009.

[4] M. Sokovic and D. Pavletic, "Quality Improvement - PDCA Cycle vs. DMAIC and DFSS," Journal of Mechanical Engineering, vol. 53, no. 6, pp. 369-378, 2007.

[5] F. T. Anbari, "Six Sigma method and its applications in project management," in Project Management Institute Annual Seminars \& Symposium, San Antonio, TX, 2002.

[6] Construction Industry Institute, CII Best Practices Handbook, Vols. 166-4, Austin, TX: Construction Industry Institute, 2017.

[7] N. Stamatiadis, P. Goodrum, E. Shocklee, R. Sturgill and C. Wang, "Tools for Applying Constructability Concepts to Project Development (Design)," University of Kentucky, 2013.

[8] P. S. Fong and J. C. Yip, "An Investigative Study of the Application of Lessons Learned Systems in Construction Projects," Journal for Education in the Built Environment, vol. 1, no. 2, pp. 27-38, 2006. https://doi.org/10.11120/jebe.2006.01020027

[9] G. Gibson Jr., C. Caldas, A. Yohe and R. Weerasooriya, "An Analysis of Lessons Learned Programs in the Construction Industry," 2008.

[10] B. G. McCullouch and R. Patty, "An INDOT Lessons Learned Cconstructability Program And Integradted Multimedia System," 1994.

[11] P. M. Goodrum, M. F. Yasin and D. E. Hancher, "Lessons Learned System for Kentucky Transportation Projects," 2003.

[12] T. Rancour and M. McCracken, "Applying 6 Sigma methods for Breakthrough Safety Performance," Professional Safety, vol. 45, no. 10, pp. 29-32, 2000.

[13] K. Yang and B. El-Haik, Design For Six Sigma: A Roadmap for Product Development, McGraw-Hill, 2003.

[14] C. B. Tayntor, Six Sigma Software Development, New York: Auerbach Publications, 2007. 